Résumés des conférences et travaux

\title{
Linguistique générale
}

\section{Claude Hagège}

\section{OpenEdition \\ Journals}

Édition électronique

URL : https://journals.openedition.org/ashp/1101

DOI : 10.4000/ashp.1101

ISSN : 1969-6310

Éditeur

Publications de l'École Pratique des Hautes Études

\section{Édition imprimée}

Date de publication : 1 octobre 2011

Pagination : 288-289

ISSN : 0766-0677

\section{Référence électronique}

Claude Hagège, "Linguistique générale », Annuaire de l'École pratique des hautes études (EPHE), Section des sciences historiques et philologiques [En ligne], 142 | 2011, mis en ligne le 18 juillet 2011, consulté le 06 juillet 2021. URL : http://journals.openedition.org/ashp/1101; DOI : https://doi.org/10.4000/ashp. 1101 


\title{
LINGUISTIQUE GÉNÉRALE
}

\author{
Directeur d'études : M. Claude HagÈGE
}

Programme de l'année 2008-2009 : La relation entre syntaxe et sémantique à la lumière de la typologie des langues.

Programme de l'année 2009-2010 : Diversité des fonctions du langage et des langues.

Les conférences des années 2008-2009 et 2009-2010 ont été consacrées au problème de la méthode par laquelle on établit des catégories de langues en linguistique générale. On a donc étudié les enseignements que l'on peut tirer d'une théorie de la catégorie de langue. Pour illustrer cette démarche, on a privilégié l'examen des instruments utilisés par la plupart des langues pour marquer les fonctions, à savoir cette catégorie de la grammaire, mais aussi du lexique, qui, selon le type de langues et l'ordre des mots qui prévaut dans la syntaxe de chaque langue, est appelée « préposition » ou « postposition » (pour s'en tenir aux réalisations les plus courantes).

Il est apparu qu'une désignation pratique était celle d' " adposition », laquelle retient, comme les deux termes de «préposition » et « postposition », le critère de la position occupée par les représentants de cette catégorie vis-à-vis du terme qu'ils régissent et mettent en relation avec le reste de l'énoncé. D'autres termes sont possibles, et se trouvent employés dans la littérature linguistique. L'un est celui de « relateur », qui dit clairement que les outils en question sont des instruments de relation, mais présente l'inconvénient de ne pas être très usuel, du moins en dehors de certains emplois d'écoles. L'autre est celui de « (mot) fonctionnel» ou d' « indicateur de fonction », qui serait adéquat, s'il n'était un terme qui parle de la fonction, mais ne dit rien de la position, critère formel essentiel. C'est pourquoi « adposition », qui subsume sous une même appellation les deux termes en « -position », et qui a, en outre, l'avantage d'être répandu parmi les typologistes, paraît la désignation la moins inadéquate.

On a consacré un long moment à délimiter les adpositions et les affixes casuels, qui, sous prétexte d'équivalence fonctionnelle, sont le plus souvent traités ensemble, sans effort pour faire apparaître leurs différences, sur lesquelles, au contraire, on a insisté. On a consacré un autre moment à la relation entre les adpositions et un certain nombre d'autres catégories de mots avec lesquelles elles sont souvent confondues, et que divers critères font apparaître, cependant, comme distinctes : il s'agit des préverbes, des indicateurs de directions, des morphèmes qui marquent une relation syntaxique directe ou inverse, des radicaux locatifs de certaines langues, des applicatifs, de certains adverbes, des morphèmes dé-prédicatifs, des modificateurs sémantiques, des topicalisateurs, des coordonnants, et enfin d'une catégorie particulière de mots qui n'ont pas, jusqu'ici, été aperçus, et qu'on propose d'appeler « chorophoriques » ou spécificateurs d'emplacement.

Enfin, on a examiné les adpositions du point de vue de leurs relations avec un type de mots qui, comme elles dans leur association avec le terme qu'elles gouvernent, 
expriment un complément circonstanciel, mais ne sont pas, contrairement à elles, des mots régissants.

L'étude syntaxique du phénomène des adpositions a été reportée à l'année prochaine, ainsi que l'étude sémantique, qui pourrait occuper aussi l'année 2011-2012. 